\title{
Phase resetting in human auditory cortex to visual speech
}

Pierre Mégevand ${ }^{1,2}$, Manuel R. Mercier ${ }^{3,4,5}$, David M. Groppe ${ }^{1,6}$, Elana Zion Golumbic ${ }^{7}$, Nima Mesgarani ${ }^{8}$, Michael S. Beauchamp ${ }^{9}$, Charles E. Schroeder ${ }^{10,11,12^{*}}$, Ashesh D. Mehta ${ }^{1,12^{*}}$

${ }^{1}$ Department of Neurosurgery, Donald and Barbara Zucker School of Medicine at Hofstra/Northwell, and Feinstein Institute for Medical Research, Manhasset, NY 11030, USA

${ }^{2}$ Department of Basic Neurosciences, Faculty of Medicine, University of Geneva, 1211 Geneva, Switzerland

${ }^{3}$ Department of Neurology, Montefiore Medical Center, Bronx, NY 10467, USA

${ }^{4}$ Department of Neuroscience, Albert Einstein College of Medicine, Bronx, NY 10461, USA

${ }^{5}$ Aix Marseille University, Inserm, Institut de Neurosciences des Systèmes, 13005 Marseille, France

${ }^{6}$ The Krembil Neuroscience Centre, University Health Network, Toronto, ON M5T 1M8, Canada

${ }^{7}$ The Gonda Brain Research Center, Bar Ilan University, Ramat Gan, 5290002, Israel

${ }^{8}$ Department of Electrical Engineering, Columbia University, New York, NY 10027, USA

${ }^{9}$ Department of Neurosurgery, Baylor College of Medicine, Houston, TX 77030, USA

${ }^{10}$ Nathan S. Kline Institute, Orangeburg, NY 10962, USA

${ }^{11}$ Department of Neurosurgery, Columbia University, New York, NY 10032, USA

${ }^{12}$ These senior authors contributed equally

*Correspondence: cs2388@columbia.edu; amehta@northwell.edu 
Running title: phase reset in auditory cortex to visual speech

Item count: 21 pages, 4 figures, 0 table, 0 multimedia, 0 3D model

Word count: 141 in abstract, 642 in introduction, 855 in discussion

\section{CONFLICT OF INTEREST STATEMENT}

The authors declare no competing financial interests.

\section{ACKNOWLEDGEMENTS}

We thank the patients for their participation, and Erin Yeagle, Willie Walker Jr., the physicians and other professionals of the Neurosurgery and Neurology departments of North Shore University Hospital, Itzik Norman and Bahar Khalighinejad for their assistance. Part of the computations for this work were performed at the University of Geneva on the Baobab cluster. This work was supported by the Swiss National Science Foundation (grants 139829, 148388 and 167836 to PM), the NINDS (NS098976 to CES, MSB and ADM) and the Page and Otto Marx Jr. Foundation to ADM.

\section{AUTHOR CONTRIBUTIONS}

Designed research: PM, EZG, CES and ADM; performed research: PM; contributed software: PM, MRM, DMG and NM; analyzed data: PM, MRM, DMG and NM; wrote the paper: PM, MSB, CES and ADM. 


\section{ABSTRACT}

Natural conversation is multisensory: when we can see the speaker's face, visual speech cues influence our perception of what is being said. The neuronal basis of this phenomenon remains unclear, though there is indication that phase modulation of neuronal oscillations-ongoing excitability fluctuations of neuronal populations in the brain-provides a mechanistic contribution. Investigating this question using naturalistic audiovisual speech with intracranial recordings in humans, we show that neuronal populations in auditory cortex track the temporal dynamics of unisensory visual speech using the phase of their slow oscillations and phase-related modulations in high-frequency activity. Auditory cortex thus builds a representation of the speech stream's envelope based on visual speech alone, at least in part by resetting the phase of its ongoing oscillations. Phase reset could amplify the representation of the speech stream and organize the information contained in neuronal activity patterns.

\section{SIGNIFICANCE STATEMENT}

Watching the speaker can facilitate our understanding of what is being said. The mechanisms responsible for this influence of visual cues on the processing of speech remain incompletely understood. We studied those mechanisms by recording the human brain's electrical activity through electrodes implanted surgically inside the skull. We found that some regions of cerebral cortex that process auditory speech also respond to visual speech even when it is shown as a silent movie without a soundtrack. This response can occur through a reset of the phase of ongoing oscillations, which helps augment the response of auditory cortex to audiovisual speech. Our results contribute to discover the mechanisms by which the brain merges auditory and visual speech into a unitary perception. 
bioRxiv preprint doi: https://doi.org/10.1101/405597; this version posted April 13,2020. The copyright holder for this preprint (which was not certified by peer review) is the author/funder, who has granted bioRxiv a license to display the preprint in perpetuity. It is made available under aCC-BY 4.0 International license.

\section{INTRODUCTION}

Viewing one's interlocutor significantly improves intelligibility under noisy conditions (Sumby and Pollack, 1954). Moreover, mismatched auditory and visual speech cues can create striking illusions (McGurk and Macdonald, 1976). Despite the ubiquity and power of visual influences on speech perception, the underlying neuronal mechanisms remain unclear. The cerebral processing of auditory and visual speech converges in multisensory cortical areas, especially the superior temporal cortex (Miller and D'Esposito, 2005; Beauchamp et al., 2010). Crossmodal influences are also found in cortex traditionally considered to be unisensory; in particular, visual speech modulates the activity of auditory cortex (Calvert et al., 1997; Besle et al., 2008; Kayser et al., 2008).

The articulatory movements that constitute visual speech strongly correlate with the corresponding speech sounds (Chandrasekaran et al., 2009; Schwartz and Savariaux, 2014; Park et al., 2016) and predict them to some extent (Arnal et al., 2009), suggesting that visual speech might serve as an alerting cue to auditory cortex, preparing the neural circuits to process the incoming speech sounds more efficiently. Earlier, we raised the hypothesis that this preparation occurs in part through a resetting of the phase of neuronal oscillations in auditory cortex: through this phase reset, visual speech cues influence the temporal pattern of neuronal excitability fluctuations in auditory cortex (Schroeder et al., 2008). This hypothesis rests on four lines of evidence. First, auditory speech has predictable rhythms, with syllables arriving at a relatively rapid rate $(4-7 \mathrm{~Hz})$ nested within the slower (1-3 Hz) rates of phrase and word production. These rhythmic features of speech are critical for it to be intelligible (Shannon et al., 1995; Greenberg et al., 2003). Second, auditory cortex synchronizes its oscillations to the rhythm of heard speech, and the magnitude of this synchronization correlates with the intelligibility of speech (Ahissar et al., 2001; Luo and Poeppel, 2007; Ding and Simon, 2014; Vander Ghinst et al., 2016). Third, neuronal oscillations correspond to momentary changes in neuronal excitability, so that independent of modality, the response of sensory cortex depends on the phase of its oscillations upon stimulus arrival (Lakatos et al., 2008). Fourth, even at the level of primary sensory cortex, oscillations can be phase-reset by stimuli from other modalities, and this crossmodal reset influences the processing of incoming stimuli from the preferred modality (Lakatos et al., 2007; Kayser et al., 2008).

Human EEG and MEG studies of cerebral responses to continuous, naturalistic audiovisual speech have established that oscillations are influenced by the visual as well as the auditory component of speech (Luo et al., 2010; Crosse et al., 2015, 2016; O'Sullivan et al., 2016; Park et al., 2016, 2018; Giordano et al., 2017). While these observations are compatible with the phase reset hypothesis, they do not rule 
out the possibility that the apparent phase alignment simply reflects a succession of crossmodal sensory-evoked responses; in fact, some favor this interpretation (Crosse et al., 2015, 2016). Our perspective is that phase-reset and evoked-response mechanisms ordinarily operate in a complementary fashion (Schroeder and Lakatos, 2009). Thus, in the present context, we expect that both will mediate visual influences on auditory speech processing. In order to dissect these influences, one must be able to resolve the local activity of a given cortical area well enough to dissociate a momentary increase in phase alignment from any coincident increase in oscillatory power (Makeig et al., 2004; Shah et al., 2004). No non-invasive neurophysiological study to date meets that standard, but invasive techniques are better suited for that level of granularity.

Here, we used intracranial EEG to probe the mechanistic basis for the effect of visual speech on auditory processing. We show that unisensory visual speech resets the phase of low-frequency neuronal oscillations in auditory cortex, helping to shape the neuronal responses of auditory cortex to visual speech. Our results strongly support crossmodal phase reset as one of the neuronal mechanisms underlying multisensory integration in audiovisual speech processing.

\section{MATERIALS AND METHODS}

\section{Experimental design}

\section{Participants}

Nine patients ( 5 women, age range 21-52 years old) suffering from drug-resistant focal epilepsy and undergoing video-intracranial EEG (iEEG) monitoring at North Shore University Hospital (Manhasset, NY 11030, USA) participated in the experiments. All participants were fluent English speakers. The participants provided written informed consent under the guidelines of the Declaration of Helsinki, as monitored by the Feinstein Institute for Medical Research's institutional review board.

\section{$\underline{\text { Stimuli and task }}$}

Stimuli (Zion Golumbic et al., 2013) were presented at the bedside using a laptop computer and Presentation software (version 17.2, Neurobehavioral Systems, Inc., Berkeley, CA; RRID: SCR_002521; http://www.neurobs.com). Trials started with a 1-s fixation cross on a black screen. The participants then viewed or heard video clips (7-12 seconds) of a speaker telling a short story. The clips were cut off to leave out the last word. A written word was then presented on the screen, and the participants had to select whether that word ended the story appropriately or not. There was no time limit for participants to indicate their answer; reaction time was not monitored. There were 2 speakers (one woman) telling 4 stories each ( 8 distinct stories); each story was presented once with one of 8 different 
ending words ( 4 appropriate), for a total of 64 trials. These were presented once in each of 3 sensory modalities: audiovisual (movie with audio track), auditory (soundtrack with a fixation cross on a black screen), visual (silent movie). Trial order was randomized, with the constraint that the same story could not be presented twice in a row, regardless of modality. Precise timing of stimulus presentation with respect to $\mathrm{iEEG}$ data acquisition was verified using an oscilloscope, a microphone and a photodiode.

The task was intended to ensure that participants were attending the stimuli. Performance was on average $85 \%$ (range 59-95\%) in the audiovisual modality, 84\% (61-95\%) in the auditory modality, and $68 \%(44-88 \%)$ in the visual modality. Performance was significantly above chance in each modality (paired t-tests; $\mathrm{AV}: \mathrm{t}(8)=8.37, \mathrm{p}=4.74 * 10^{-5} ; \mathrm{A}: \mathrm{t}(8)=8.81, \mathrm{p}=4.74 * 10^{-5} ; \mathrm{V}: \mathrm{t}(8)=3.44, \mathrm{p}=0.0088 ; \mathrm{p}$-values corrected for multiple comparisons using the false-discovery rate procedure (Benjamini and Hochberg, 1995)).

\section{Data acquisition}

\section{iEEG electrode localization}

The placement of iEEG electrodes (subdural and depth electrodes, Ad-Tech Medical, Racine, WI, and Integra LifeSciences, Plainsboro, NJ) was determined on clinical grounds, without reference to this study. The localization and display of iEEG electrodes was performed using iELVis (RRID: SCR_016109; http://ielvis.pbworks.com) (Groppe et al., 2017). For each participant, a post-implantation highresolution CT scan was coregistered with a post-implantation 3D T1 1.5-tesla MRI scan and then with a pre-implantation 3D T1 3-tesla MRI scan via affine transforms with 6 degrees of freedom using the FMRIB Linear Image Registration Tool included in the FMRIB Software Library (RRID: SCR_002823; https://fsl.fmrib.ox.ac.uk/fsl/fslwiki) (Jenkinson et al., 2012) or the bbregister tool included in FreeSurfer (RRID: SCR_001847; https://surfer.nmr.mgh.harvard.edu/fswiki/FreeSurferWiki) (Fischl, 2012). Electrodes were localized manually on the CT scan using Biolmage Suite (RRID: SCR_002986; http://bioimagesuite.yale.edu/) (Papademetris et al., 2006). The pre-implantation 3D T1 MRI scan was processed using FreeSurfer to segment the white matter, deep grey matter structures, and cortex, reconstruct the pial surface, approximate the leptomeningeal surface (Schaer et al., 2008), and parcellate the neocortex according to gyral anatomy (Desikan et al., 2006). In order to compensate for the brain shift that accompanies the insertion of subdural electrodes through a large craniotomy, subdural electrodes were projected back to the pre-implantation leptomeningeal surface (Dykstra et al., 2012). 
bioRxiv preprint doi: https://doi.org/10.1101/405597; this version posted April 13,2020. The copyright holder for this preprint (which was not certified by peer review) is the author/funder, who has granted bioRxiv a license to display the preprint in perpetuity. It is made available under aCC-BY 4.0 International license.

\section{iEEG recording and preprocessing}

Intracranial EEG signals were referenced to a vertex subdermal electrode, filtered and digitized (0.1 $\mathrm{Hz}$ high-pass filter, $200 \mathrm{~Hz}$ low-pass filter, 500-512 samples per second, XLTEK EMU128FS or Natus Neurolink IP 256 systems, Natus Medical, Inc., Pleasanton, CA). Analysis was performed offline using the FieldTrip toolbox (RRID: SCR_004849; http://www.fieldtriptoolbox.org/) (Oostenveld et al., 2011) and custom-made programs for MATLAB (The MathWorks Inc., Natick, MA; RRID: SCR_001622; https://www.mathworks.com/products/matlab.html). 60- $\mathrm{Hz}$ line noise and its harmonics were filtered out using a discrete Fourier transform filter. iEEG electrodes contaminated with noise or abundant epileptiform activity were identified visually and rejected. iEEG electrodes that lay in white matter were also rejected (Mercier et al., 2017). The remaining iEEG signals were re-referenced to average reference.

The absolute phase and power of an iEEG signal are quantities that depend on the reference; consequently, the quantification of synchronization between electrodes is strongly influenced by the choice of the reference (Guevara et al., 2005; Mercier et al., 2017). Here, however, we strictly focus on the relative relationship between a continuous sensory stimulus and the phase or power response at a given electrode; therefore, at no point is the phase or power of an iEEG signal measured at a given electrode compared to those at another. Furthermore, all statistical testing is performed at the singleelectrode level through permutation testing; thus, any influence of the reference on the observed data is also present in the surrogate data generated by the permutation test (see below). For these reasons, the analyses presented here are immune to the choice of a particular referencing scheme.

\section{Data analysis}

$\underline{\text { Time courses of auditory and visual speech stimuli }}$

The envelope of auditory speech stimuli was computed by filtering the audio track of the video clips through a gammatone filter bank approximating a cochlear filter, with 128 center frequencies equally spaced on the equivalent rectangle bandwidth-rate scale and ranging from 80 and $5000 \mathrm{~Hz}$ (Carney and Yin, 1988), computing the Hilbert transform to obtain power in each frequency band, and averaging again over frequencies (University of Surrey's Institute of Sound Recording MATLAB Toolbox; https://github.com/loSR-Surrey/MatlabToolbox). The time course of visual speech stimuli was estimated by manually measuring the vertical opening of the mouth on each still frame of the video clips (Park et al., 2016). Auditory and visual speech stimulus time courses were then resampled to $200 \mathrm{~Hz}$ at the same time points as the iEEG signals. 


\section{$\underline{\text { Time-frequency analysis of iEEG signals }}$}

To obtain instantaneous low-frequency power and phase, the iEEG signal was filtered between 0.5 and $9 \mathrm{~Hz}$ (6th-order Butterworth filters), downsampled to $200 \mathrm{~Hz}$, and Hilbert-transformed. Broadband high-frequency activity (BHA), which reflects local neuronal activity (Crone et al., 1998; Ray et al., 2008), was computed by filtering the iEEG signal in 10-Hz bands between 75 and $175 \mathrm{~Hz}$ (4thorder Butterworth filters), computing the Hilbert transform to obtain instantaneous power, dividing instantaneous power in each band by its own mean over time in order to compensate for the $1 / \mathrm{f}$ power drop, and then averaging again over bands (Golan et al., 2016). BHA was then downsampled to $200 \mathrm{~Hz}$.

\section{$\underline{\text { Stimulus-response cross-correlation }}$}

The relationship between speech stimuli and brain responses was quantified by computing their crosscorrelation. For each iEEG electrode, data from all trials in each sensory modality were concatenated and were then cross-correlated with the corresponding concatenated stimulus time courses. For lowfrequency power and BHA, Pearson correlation was computed; for low-frequency phase, linear-tocircular correlation was computed (Berens, 2009). In order to account for the fact that brain responses to sensory stimuli occur with some delay, lags of -200 to $+200 \mathrm{~ms}$ between stimuli and responses were allowed. The maximum of the absolute value of the correlation coefficient over this time period was considered.

\section{Statistical testing}

In order to assess the statistical significance of observed correlation coefficients, their distribution under the null hypothesis was estimated using a permutation test. In each iteration, trial labels were shuffled to disrupt the temporal relationship between stimuli and responses, and one value of the correlation coefficient was computed. The procedure was repeated 1000 times. Observed values of correlation coefficients were then expressed as z-scores of the null distribution. The procedure is illustrated in Figure 2A-E.

\section{Correction for multiple comparisons}

P-values were corrected for multiple comparisons over electrodes using a false discovery rate (FDR) procedure (Benjamini and Hochberg, 1995) with family-wise error rate set at 0.05 , implemented in the Mass Univariate ERP toolbox (RRID: SCR_016108; https://github.com/dmgroppe/Mass Univariate ERP Toolbox) (Groppe et al., 2011). The Benjamini- 
bioRxiv preprint doi: https://doi org/10.1101/405597; this version posted April 13, 2020. The copyright holder for this preprint (which was not certified by peer review) is the author/funder, who has granted bioRxiv a license to display the preprint in perpetuity. It is made available under aCC-BY 4.0 International license.

Hochberg FDR procedure maintains adequate control of the family-wise error rate also in the case of positive dependencies between the observed variables.

\section{Data and software availability}

Data and custom-made software are available upon request from Pierre Mégevand (pierre.megevand@unige.ch).

\section{RESULTS}

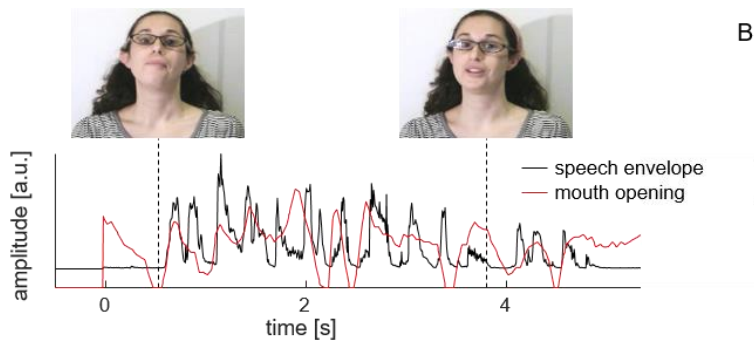

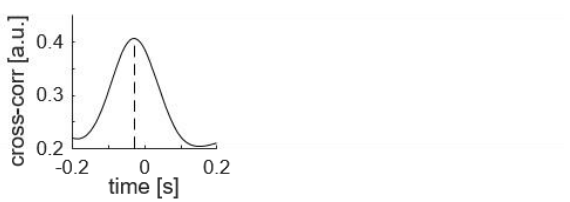
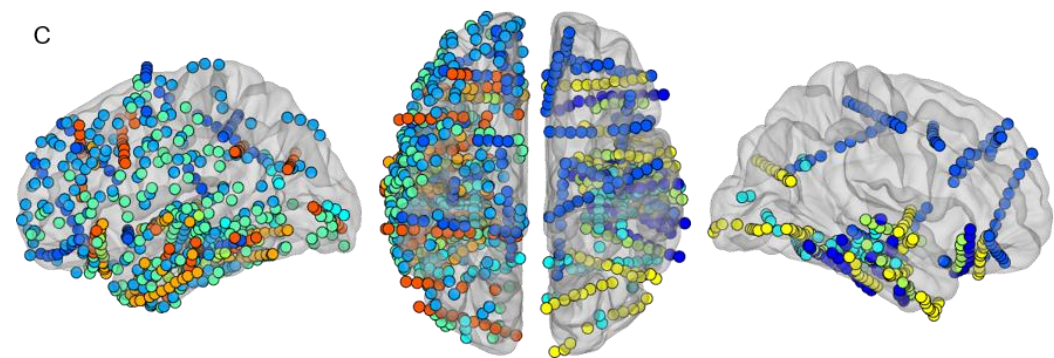

Figure 1. Speech stimuli and iEEG electrode coverage. A. Speech stimuli are approximately 10-second-long stories presented either in both auditory and visual modalities, auditory-only, or visual-only. The sound envelope of speech and the extent of vertical mouth opening are plotted for one story fragment. B. Over all stories, there is a 30-ms lead of mouth movements over the speech envelope.

C. All cortical sites included in the study $(\mathrm{N}=1012)$ are plotted on a semi-transparent template brain, color-coded for each of the 9 patients. Left: lateral view of the left hemisphere; center: superior view of both hemispheres (left hemisphere on the left, frontal pole at the top, occipital pole at the bottom); right: lateral view of the right hemisphere.

\section{Cortical tracking of auditory speech}

We recorded intracranial EEG (iEEG) signals from electrodes implanted in the brain of nine human participants undergoing invasive electrophysiological monitoring for epilepsy (Fig. 1C). Patients attended to clips of a speaker telling a short story, presented in the auditory (soundtrack with black screen), visual (silent movie) and audiovisual modalities (Fig. 1A). Cortical sites were considered to be auditory-responsive if the time course of their local neuronal activity, assessed by broadband highfrequency activity (BHA, also known as "high-gamma power") (Crone et al., 1998), correlated 
bioRxiv preprint doi: https://doi.org/10.1101/405597; this version posted April 13,2020. The copyright holder for this preprint (which was not certified by peer review) is the author/funder, who has granted bioRxiv a license to display the preprint in perpetuity. It is made available under aCC-BY 4.0 International license.

significantly with that of auditory speech (indexed by the amplitude of the speech envelope). We quantified the magnitude of speech-brain correlations through cross-correlation and tested for significance using permutation testing, as illustrated in Figure 2A-E. 186 cortical sites, centered mostly on the superior and middle temporal gyri of both cerebral hemispheres, displayed significant BHA tracking of auditory speech (Fig. 2F). These sites were analyzed further as auditory-responsive cortex.

A

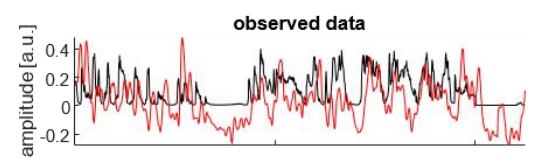

C
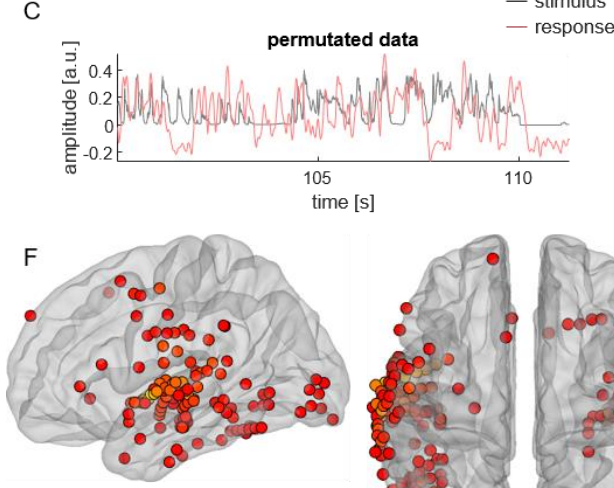

z-score
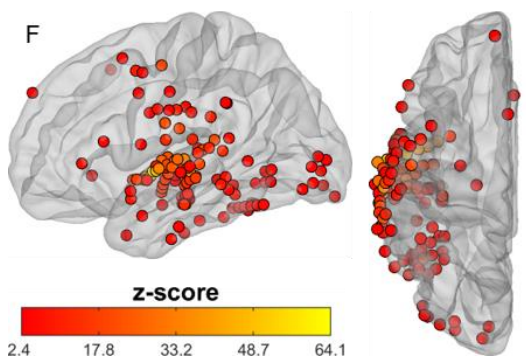

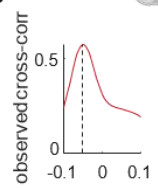

D
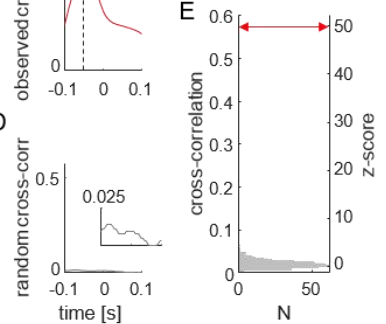
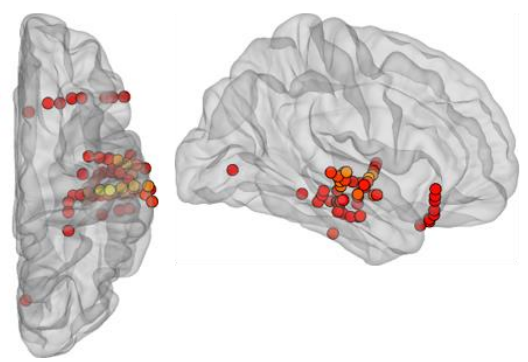

Figure 2. Establishing correlations between speech stimuli and cortical responses. A. In this example cortical site, located in the left superior temporal gyrus (inset at top right), broadband high-frequency activity (BHA, red trace) closely tracks the sound envelope of speech (black trace). B. Cortical tracking of speech is quantified by computing the maximum cross-correlation between stimulus and response. Here, cross-correlation reaches a maximum at $-100 \mathrm{~ms}$, the negative value indicating that the brain response lags behind the sensory stimulus). C. In order to assess to what extent the observed cortical tracking of speech departs from the null hypothesis, the trial labels of responses are permutated at random so that they are no longer aligned with the corresponding stimuli. D. A random cross-correlation is computed in the same fashion as the observed one. Inset: zoom on the y-axis. E. The permutation procedure is repeated 1000 times, yielding a distribution of cross-correlation values under the null hypothesis (gray histogram). The observed cross-correlation value (red arrow) is expressed as a $z$-score of that null distribution (here $z=49.5)$. F. Applying this procedure to the entire dataset, 186 of $1012(18 \%)$ cortical sites display significant tracking of the sound envelope of speech with their BHA at the $p<=0.05$ level, FDR-corrected over all sites. These sites are selected as auditory-responsive cortex.

We also examined how low-frequency activity in auditory-responsive cortex tracks auditory speech. As expected, we found very strong tracking through both low-frequency phase and power, the intensity of which correlated strongly with BHA tracking (Fig. 3A-B). Next, we asked whether the 
bioRxiv preprint doi: https://doi.org/10.1101/405597; this version posted April 13, 2020. The copyright holder for this preprint (which was not certified by peer review) is the author/funder, who has granted bioRxiv a license to display the preprint in perpetuity. It is made available under aCC-BY 4.0 International license.

tracking of the speech envelope differed in response to audiovisual speech compared to unisensory auditory speech. Tracking through the phase of low-frequency activity was stronger for audiovisual speech than for purely auditory speech, whereas tracking through low-frequency power was weaker for audiovisual than for auditory speech (Fig. 3C). The improvement in phase tracking in the audiovisual condition suggests that visual speech signals provide an additional influence to auditory cortex, which improves the phase alignment of its low-frequency activity to the speech envelope. The appearance of the opposite trend in low-frequency power tracking is inconsistent with the idea that this improvement is simply an artifact of increased evoked-response power.
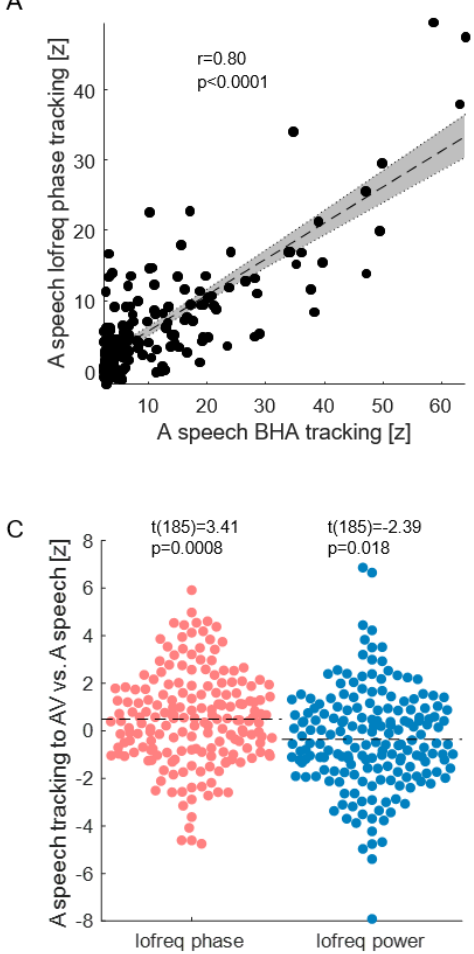

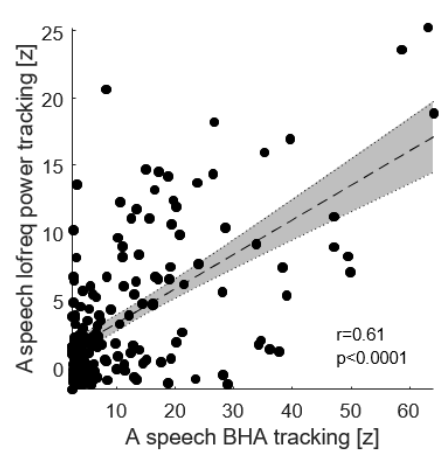

Figure 3. Low-frequency cortical tracking of auditory and audiovisual speech. A. Auditory-responsive cortex tracks the speech envelope through both its BHA and the phase of its low-frequency $(0.5-9 \mathrm{~Hz}) \mathrm{EEG}$ activity, and the two metrics are strongly correlated (Pearson correlation, $n=186)$. B. Auditory-responsive cortex also tracks the speech envelope through the power of its low-frequency oscillations (Pearson correlation, $n=186$ ). C. The intensity of speech tracking by low-frequency phase (red) is greater in response to audiovisual (AV) than unisensory auditory $(A)$ speech (paired t-test). Conversely, the intensity of speech tracking by low-frequency power (blue) is reduced in response to AV compared to A speech.

\section{Tracking of visual speech by auditory-responsive cortex}

We then asked how unisensory visual speech influences low-frequency activity in auditory-responsive cortex. To index the time course of visual speech, we measured the vertical opening of the mouth, a metric that correlates with the area of mouth opening and with the speech envelope (see Fig. 1B). We 
bioRxiv preprint doi: https://doi org/10.1101/405597; this version posted April 13, 2020. The copyright holder for this preprint (which was not certified by peer review) is the author/funder, who has granted bioRxiv a license to display the preprint in perpetuity. It is made available under aCC-BY 4.0 International license.

quantified the intensity of tracking of mouth opening by either low-frequency phase or power in auditory-responsive cortex, using the same approach as for the tracking of the speech envelope. We found that a subset of auditory-responsive cortical sites displayed phase tracking of visual speech (Fig. $4 A-B)$. These sites were focused in the superior temporal gyrus and temporo-occipital cortex. We also found power tracking of visual speech in another subset of auditory-responsive cortical sites (Fig. $4 A, C)$. Importantly, these sites were generally different from those that displayed phase tracking, and their anatomical localization was more diffuse, spreading to the inferior temporal, parietal and frontal cortices. This segregation of phase and power tracking sites is consistent with the idea that phase reset and evoked responses provide complementary mechanisms for the influence of visual speech in auditory cortex.
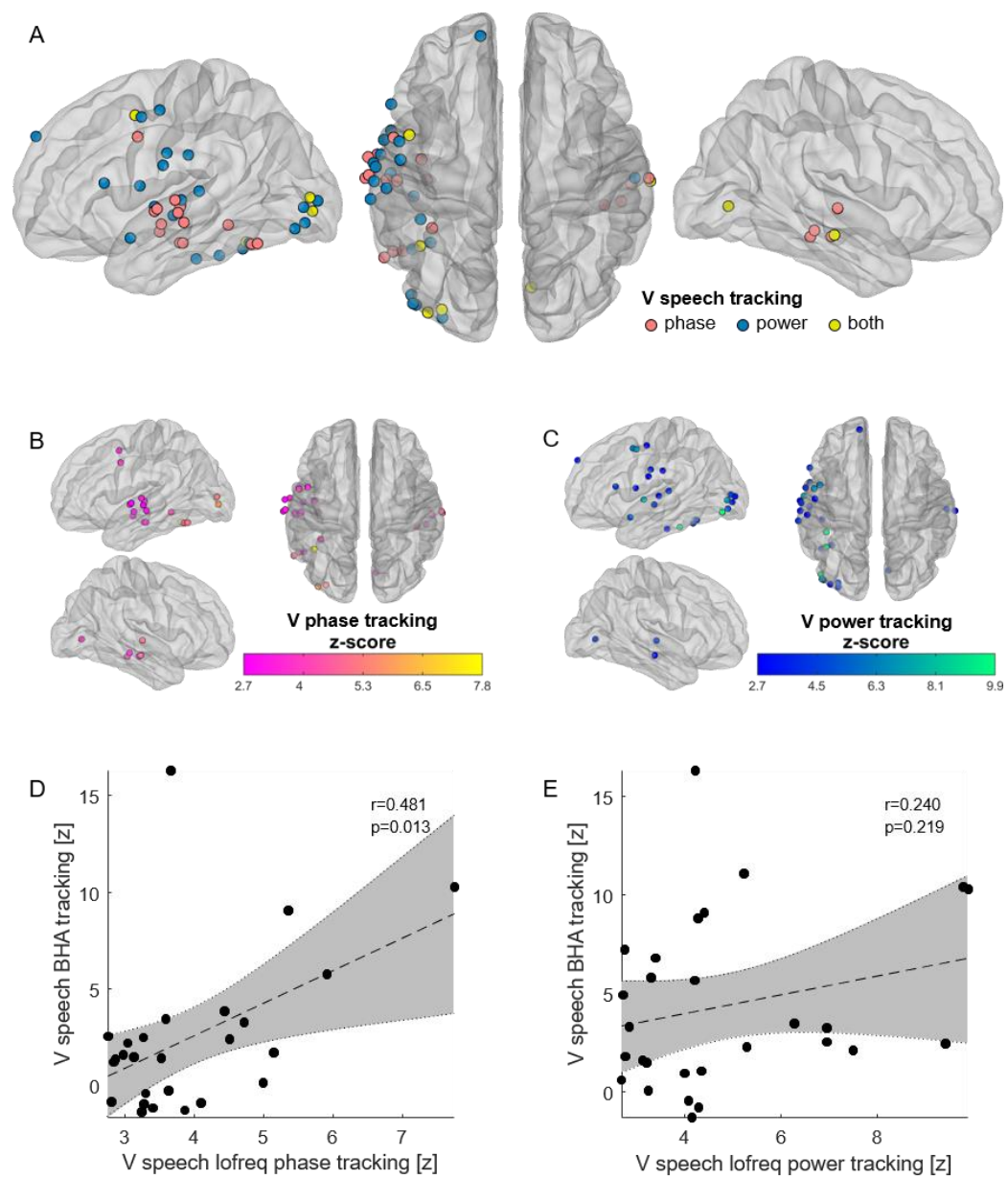

Figure 4. Low-frequency tracking of visual speech in auditory-responsive cortex. A. Tracking of visual speech (the temporal pattern of vertical mouth opening) by low-frequency (0.5-9 Hz) iEEG activity at auditory-responsive cortical sites. Sites that display phase tracking $(n=26)$ are plotted in red, sites that display power tracking $(n=28)$ in blue, and sites that display both $(n=6)$ in yellow. Significance is determined at the $p_{F D R}<=0.05$ level, corrected over all 1012 sites. The number of sites that display both phase and power tracking is not higher than expected by chance, given the number of sites displaying either and the total number of $\operatorname{sites}(z=1.24, p=0.11$, permutation 
test). B. The intensity of low-frequency phase tracking of visual speech by auditory-responsive sites is colorcoded. C. The intensity of low-frequency power tracking of visual speech by auditory-responsive sites is colorcoded. D. In the sites that track visual speech with low-frequency phase, there is a correlation between that tracking and tracking with BHA (Pearson correlation). E. By contrast, there is no correlation between lowfrequency power tracking and BHA tracking of visual speech.

Lastly, we examined the influence of phase reset on local neuronal activation as indexed by BHS. The intensity of BHA tracking correlated with that of tracking through low-frequency phase (Fig. 4D), indicating coupling between low-frequency phase and the amplitude of neuronal activation (Canolty et al., 2009). By contrast, there was no detectable correlation between BHA tracking and lowfrequency power tracking (Fig. 4E). These observations are consistent with the hypothesis that phase reset to visual speech augments local neuronal activation in auditory cortex.

\section{DISCUSSION}

It is widely observed that both phase-entrained low-frequency activity and fluctuations in broadband high-frequency activity in auditory cortex track the temporal dynamics of unisensory auditory speech (see (Ding and Simon, 2014) for a review). It is also hypothesized that visual speech gestures contribute to intelligibility by facilitating auditory cortical entrainment to the speech stream (Schroeder et al., 2008). Non-invasive neurophysiological studies have shown that the visual component of audiovisual speech influences cerebral activity, mostly in visual areas, but also in superior temporal, inferior frontal and premotor cortex (Luo et al., 2010; Park et al., 2016, 2018; Giordano et al., 2017). They have also detailed interactions between brain regions that are critical for audiovisual speech perception (Park et al., 2016, 2018). Furthermore, the reconstruction of the temporal dynamics of speech is more accurate from whole-brain responses to audiovisual speech than to unisensory auditory speech, and it is also possible to perform this reconstruction from whole-brain responses to unisensory visual speech (Crosse et al., 2015, 2016; O'Sullivan et al., 2016). Collectively, these studies demonstrate that cortical dynamics align to visual speech, but do not reveal the underlying neuronal mechanisms. Here, we used $\mathrm{BEEG}$ recordings for a more direct examination of the neurophysiological mechanisms underlying visual enhancement of auditory cortical speech processing. Our findings significantly elaborate the mechanistic description of crossmodal stimulus processing as a critical contribution to speech perception under complex and noisy natural conditions.

Three of our findings summarize the impact of this study. First, within the cortical network that responds to auditory speech, tracking by low-frequency phase is enhanced by audiovisual compared to auditory-alone stimulation, while the opposite is true for speech tracking by power fluctuations in 
bioRxiv preprint doi: https://doi.org/10.1101/405597; this version posted April 13, 2020. The copyright holder for this preprint (which was not certified by peer review) is the author/funder, who has granted bioRxiv a license to display the preprint in perpetuity. It is made available under aCC-BY 4.0 International license.

the same low-frequency band (Fig. 3). As noted above, this dissociation is inconsistent with the idea that the enhancement of phase tracking in the audiovisual condition is simply an artifact of increased evoked-response power. The dissociation may also help to explain an often-noted paradox: despite the general perceptual amplification that attends audiovisual speech, neurophysiological responses to audiovisual stimuli in both auditory and visual cortex are generally smaller than those to the preferred-modality stimulus alone (Besle et al., 2008; Mercier et al., 2013, 2015; Schepers et al., 2014). Second, we observed an anatomical dissociation between the sites that display phase tracking of visual speech and those that display power tracking (Fig. 4). This segregation of phase and power tracking sites is consistent with the idea that phase-reset/entrainment and evoked responses provide complementary mechanisms for the influence of visual speech in auditory cortex. Finally, the magnitude of visual speech tracking by BHA correlated significantly with that of tracking through lowfrequency phase, but there was no detectable correlation between BHA tracking and low-frequency power tracking (Fig. 4D-E). This pattern of effects suggests that phase reset by visual speech augments local neuronal activation in auditory cortex. Taken together, these findings support the hypothesis that oscillatory phase reset is a mechanism by which visual speech cues influence the processing of speech sounds by auditory cortex (Schroeder et al., 2008).

Previous human iEEG studies showed that the posterior superior temporal gyrus responds to both the auditory and visual components of audiovisual speech (Ozker et al., 2017; Micheli et al., 2018), but did not focus on the underlying neuronal mechanisms. In a monkey study that focused on a voice-sensitive area in the anterior temporal lobe, audiovisual vocalization stimuli that began with a visual-only component produced an initial crossmodal evoked response. The timing of that evoked response with respect to the onset of the auditory vocalization, and thus the phase of visual-evoked activity in auditory cortex, determined whether neurons increased or decreased their firing rate in response to the incoming auditory stimulus (Perrodin et al., 2015). However, that study did not demonstrate that multisensory integration of audiovisual stimuli occurs through phase reset in auditory cortex, precisely because of the initial crossmodal response evoked by the visual stimulus; i.e., any phase concentration detected at this point could be an artifact of a phasic power increase.

The pattern of rapid quasi-rhythmic phase resetting that we observe has strong implications for the mechanistic understanding of speech processing in general. Indeed, this phase resetting aligns the ambient excitability fluctuations in auditory cortex with the incoming sensory stimuli, potentially helping to parse the continuous speech stream into linguistically relevant processing units such as syllables (Schroeder et al., 2008; Giraud and Poeppel, 2012; Zion Golumbic et al., 2012). As attention 
strongly reinforces the tracking of a specific speech stream (Mesgarani and Chang, 2012; Zion Golumbic et al., 2013; O’Sullivan et al., 2015), phase resetting will tend to amplify an attended speech stream above background noise, increasing its perceptual salience.

It is clear that visual enhancement of speech takes place within the context of strong top-down influences from frontal and parietal regions that support the processing of distinct linguistic features (Park et al., 2016, 2018; Di Liberto et al., 2018; Keitel et al., 2018). It also appears that low-frequency oscillations relevant to speech perception can themselves be modulated by transcranial electrical stimulation (Zoefel et al., 2018). Our findings highlight the need to consider oscillatory phase in targeting potential neuromodulation therapy to enhance communication. 


\section{REFERENCES}

Ahissar E, Nagarajan S, Ahissar M, Protopapas A, Mahncke H, Merzenich MM (2001) Speech comprehension is correlated with temporal response patterns recorded from auditory cortex. Proc Natl Acad Sci U S A 98:13367-13372 Available at: http://www.ncbi.nlm.nih.gov/pubmed/11698688.

Arnal LH, Morillon B, Kell CA, Giraud A-L (2009) Dual Neural Routing of Visual Facilitation in Speech Processing. J Neurosci 29:13445-13453 Available at: http://www.jneurosci.org/cgi/doi/10.1523/JNEUROSCI.3194-09.2009.

Beauchamp MS, Nath AR, Pasalar S (2010) fMRI-Guided Transcranial Magnetic Stimulation Reveals That the Superior Temporal Sulcus Is a Cortical Locus of the McGurk Effect. J Neurosci 30:24142417 Available at: http://www.jneurosci.org/cgi/doi/10.1523/JNEUROSCI.4865-09.2010.

Benjamini Y, Hochberg Y (1995) Controlling the false discovery rate: a practical and powerful approach to multiple testing. J R Stat Soc 57:289-300 Available at: https://www.jstor.org/stable/2346101 [Accessed February 28, 2018].

Berens P (2009) CircStat: A MATLAB toolbox for circular statistics. J Stat Softw 31:1-21.

Besle J, Fischer C, Bidet-Caulet A, Lecaignard F, Bertrand O, Giard M-H (2008) Visual activation and audiovisual interactions in the auditory cortex during speech perception: intracranial recordings in humans. J Neurosci 28:14301-14310.

Calvert GA, Bullmore ET, Brammer MJ, Campbell R, Williams SC, McGuire PK, Woodruff PW, Iversen SD, David AS (1997) Activation of auditory cortex during silent lipreading. Science 276:593-596 Available at: http://www.sciencemag.org/cgi/doi/10.1126/science.276.5312.593.

Canolty RT, Edwards E, Dalal SS, Soltani M, Nagarajan SS, Kirsch HE, Berger MS, Barbaro NM, Knight RT (2009) High gamma power is phase-locked to theta oscillations in human neocortex. Science 313:1626-1628 Available at: http://www.pubmedcentral.nih.gov/articlerender.fcgi?artid=2628289\&tool=pmcentrez\&rende rtype=abstract\%5Cnhttp://www.sciencemag.org/cgi/doi/10.1126/science.1128115\%5Cnhttp:// www.sciencemag.org/content/313/5793/1626.short\%5Cnhttp://www.sciencemag.org/content

Carney LH, Yin TCT (1988) Temporal coding of resonances by low-frequency auditory nerve fibers: single-fiber responses and a population model. J Neurophysiol 60:1653-1677.

Chandrasekaran C, Trubanova A, Stillittano S, Caplier A, Ghazanfar AA (2009) The natural statistics of audiovisual speech. PLoS Comput Biol 5.

Crone NE, Miglioretti DL, Gordon B, Lesser RP (1998) Functional mapping of human sensorimotor cortex with electrocorticographic spectral analysis. II. Event-related synchronization in the 
gamma band. Brain 121:2301-2315.

Crosse MJ, Butler JS, Lalor EC (2015) Congruent Visual Speech Enhances Cortical Entrainment to Continuous Auditory Speech in Noise-Free Conditions. J Neurosci 35:14195-14204 Available at: http://www.jneurosci.org/content/35/42/14195.full.

Crosse MJ, Di Liberto GM, Lalor EC (2016) Eye Can Hear Clearly Now: Inverse Effectiveness in Natural Audiovisual Speech Processing Relies on Long-Term Crossmodal Temporal Integration. J Neurosci 36:9888-9895 Available at: http://www.ncbi.nlm.nih.gov/pubmed/27656026 [Accessed February 8, 2019].

Desikan RS, Ségonne F, Fischl B, Quinn BT, Dickerson BC, Blacker D, Buckner RL, Dale AM, Maguire RP, Hyman BT, Albert MS, Killiany RJ (2006) An automated labeling system for subdividing the human cerebral cortex on MRI scans into gyral based regions of interest. Neuroimage 31:968-980 Available at: http://www.ncbi.nlm.nih.gov/pubmed/16530430.

Di Liberto GM, Peter V, Kalashnikova M, Goswami U, Burnham D, Lalor EC (2018) Atypical cortical entrainment to speech in the right hemisphere underpins phonemic deficits in dyslexia. Neuroimage 175:70-79 Available at: https://doi.org/10.1016/j.neuroimage.2018.03.072.

Ding N, Simon JZ (2014) Cortical entrainment to continuous speech: functional roles and interpretations. Front Hum Neurosci 8 Available at: http://journal.frontiersin.org/article/10.3389/fnhum.2014.00311/abstract [Accessed February $5,2020]$.

Dykstra AR, Chan AM, Quinn BT, Zepeda R, Keller CJ, Cormier J, Madsen JR, Eskandar EN, Cash SS (2012) Individualized localization and cortical surface-based registration of intracranial electrodes. Neuroimage Available at: http://linkinghub.elsevier.com/retrieve/pii/S1053811911013279.

Fischl B (2012) FreeSurfer. Neuroimage 62:774-781 Available at: http://linkinghub.elsevier.com/retrieve/pii/S1053811912000389.

Giordano BL, Ince RAA, Gross J, Schyns PG, Panzeri S, Kayser C (2017) Contributions of local speech encoding and functional connectivity to audio-visual speech perception. Elife 6 Available at: https://elifesciences.org/articles/24763 [Accessed February 8, 2019].

Giraud A-L, Poeppel D (2012) Cortical oscillations and speech processing: emerging computational principles and operations. Nat Neurosci 15:511-517 Available at: http://dx.doi.org/10.1038/nn.3063.

Golan T, Davidesco I, Meshulam M, Groppe DM, Mégevand P, Yeagle EM, Goldfinger MS, Harel M, Melloni L, Schroeder CE, Deouell LY, Mehta AD, Malach R (2016) Human intracranial recordings link suppressed transients rather than "filling-in" to perceptual continuity across blinks. Elife 5:1- 
28 Available at: http://www.ncbi.nlm.nih.gov/pubmed/27685352.

Greenberg S, Carvey H, Hitchcock L, Chang S (2003) Temporal properties of spontaneous speech - A syllable-centric perspective. J Phon 31:465-485.

Groppe DM, Bickel S, Dykstra AR, Wang X, Mégevand P, Mercier MR, Lado FA, Mehta AD, Honey CJ

(2017) iELVis: An open source MATLAB toolbox for localizing and visualizing human intracranial electrode data. J Neurosci Methods 281:40-48 Available at: http://dx.doi.org/10.1016/j.jneumeth.2017.01.022.

Groppe DM, Urbach TP, Kutas M (2011) Mass univariate analysis of event-related brain potentials/fields I: A critical tutorial review. Psychophysiology 48:1711-1725.

Guevara R, Velazquez JLP, Nenadovic V, Wennberg R, Senjanović G, Dominguez LG (2005) Phase synchronization measurements using electroencephalographic recordings: What can we really say about neuronal synchrony? Neuroinformatics 3:301-313.

Jenkinson M, Beckmann CF, Behrens TEJ, Woolrich MW, Smith SM (2012) FSL. Neuroimage 62:782790 Available at: http://www.ncbi.nlm.nih.gov/pubmed/21979382.

Kayser C, Petkov Cl, Logothetis NK (2008) Visual modulation of neurons in auditory cortex. Cereb Cortex 18:1560-1574 Available at: http://www.ncbi.nlm.nih.gov/pubmed/18180245.

Keitel A, Gross J, Kayser C (2018) Perceptually relevant speech tracking in auditory and motor cortex reflects distinct linguistic features. PLoS Biol 16:1-19.

Lakatos P, Chen CM, O'Connell MN, Mills A, Schroeder CE (2007) Neuronal Oscillations and Multisensory Interaction in Primary Auditory Cortex. Neuron 53:279-292.

Lakatos P, Karmos G, Mehta ADAD, Ulbert I, Schroeder CECECE (2008) Entrainment of neuronal oscillations as a mechanism of attentional selection. Science 320:110-113 Available at: http://www.sciencemag.org/cgi/content/abstract/320/5872/110\%5Cnhttp://www.ncbi.nlm.ni h.gov/pubmed/18388295.

Luo H, Liu Z, Poeppel D (2010) Auditory cortex tracks both auditory and visual stimulus dynamics using low-frequency neuronal phase modulation. PLoS Biol 8:25-26.

Luo H, Poeppel D (2007) Phase Patterns of Neuronal Responses Reliably Discriminate Speech in Human Auditory Cortex. Neuron 54:1001-1010.

Makeig S, Debener S, Onton J, Delorme A (2004) Mining event-related brain dynamics. Trends Cogn Sci 8:204-210 Available at: https://www.sciencedirect.com/science/article/pii/S1364661304000816?via\%3Dihub [Accessed March 13, 2018].

McGurk H, Macdonald J (1976) Hearing lips and seeing voices. Nature 264:691-811.

Mercier MR, Bickel S, Megevand P, Groppe DM, Schroeder CE, Mehta AD, Lado FA (2017) Evaluation 
of cortical local field potential diffusion in stereotactic electro-encephalography recordings: A glimpse on white matter signal. Neuroimage 147:219-232 Available at: http://linkinghub.elsevier.com/retrieve/pii/S1053811916304232.

Mercier MR, Foxe JJ, Fiebelkorn IC, Butler JS, Schwartz TH, Molholm S (2013) Auditory-driven phase reset in visual cortex: Human electrocorticography reveals mechanisms of early multisensory integration. Neuroimage Available at: http://dx.doi.org/10.1016/j.neuroimage.2013.04.060.

Mercier MR, Molholm S, Fiebelkorn IC, Butler JS, Schwartz TH, Foxe JJ (2015) Neuro-oscillatory phase alignment drives speeded multisensory response times: an electro-corticographic investigation. J Neurosci 35:8546-8557 Available at: http://www.jneurosci.org/content/35/22/8546.abstract. Mesgarani N, Chang EF (2012) Selective cortical representation of attended speaker in multi-talker speech perception. Nature 485:233-236 Available at: http://www.pubmedcentral.nih.gov/articlerender.fcgi?artid=3870007\&tool=pmcentrez\&rende rtype=abstract.

Micheli C, Schepers IM, Ozker M, Yoshor D, Beauchamp MS, Rieger JW (2018) Electrocorticography reveals continuous auditory and visual speech tracking in temporal and occipital cortex. Eur J Neurosci Available at: http://doi.wiley.com/10.1111/ejn.13992 [Accessed February 8, 2019].

Miller LM, D'Esposito M (2005) Perceptual fusion and stimulus coincidence in the cross-modal integration of speech. J Neurosci 25:5884-5893 Available at: http://www.jneurosci.org/content/jneuro/25/25/5884.full.pdf [Accessed February 19, 2018].

O'Sullivan AE, Crosse MJ, Di Liberto GM, Lalor EC (2016) Visual Cortical Entrainment to Motion and Categorical Speech Features during Silent Lipreading. Front Hum Neurosci 10:679 Available at: http://journal.frontiersin.org/article/10.3389/fnhum.2016.00679/full.

O'Sullivan JA, Power AJ, Mesgarani N, Rajaram S, Foxe JJ, Shinn-Cunningham BG, Slaney M, Shamma SA, Lalor EC (2015) Attentional Selection in a Cocktail Party Environment Can Be Decoded from Single-Trial EEG. Cereb Cortex 25:1697-1706 Available at: http://www.ncbi.nlm.nih.gov/pubmed/24429136.

Oostenveld R, Fries P, Maris E, Schoffelen JM (2011) FieldTrip: Open source software for advanced analysis of MEG, EEG, and invasive electrophysiological data. Comput Intell Neurosci 2011.

Ozker M, Schepers IM, Magnotti JF, Yoshor D, Beauchamp MS (2017) A Double Dissociation between Anterior and Posterior Superior Temporal Gyrus for Processing Audiovisual Speech Demonstrated by Electrocorticography. J Cogn Neurosci 29:1044-1060 Available at: http://www.mitpressjournals.org/doi/10.1162/jocn_a_01110 [Accessed February 6, 2019]. Papademetris X, Jackowski MP, Rajeevan N, DiStasio M, Okuda H, Constable RT, Staib LH (2006) 
Biolmage Suite: An integrated medical image analysis suite: An update. Insight J 2006:209 Available at: http://www.ncbi.nlm.nih.gov/pubmed/25364771.

Park H, Ince RAA, Schyns PG, Thut G, Gross J (2018) Representational interactions during audiovisual speech entrainment: Redundancy in left posterior superior temporal gyrus and synergy in left motor cortex. Bizley J, ed. PLoS Biol 16:e2006558 Available at: https://dx.plos.org/10.1371/journal.pbio.2006558 [Accessed December 19, 2018].

Park H, Kayser C, Thut G, Gross J (2016) Lip movements entrain the observers' low-frequency brain oscillations to facilitate speech intelligibility. Elife 5:e14521 Available at: https://elifesciences.org/articles/14521 [Accessed February 27, 2018].

Ray S, Crone NE, Niebur E, Franaszczuk PJ, Hsiao SS (2008) Neural correlates of high-gamma oscillations $(60-200 \mathrm{~Hz})$ in macaque local field potentials and their potential implications in electrocorticography. J Neurosci 28:11526-11536.

Schaer M, Bach Cuadra M, Tamarit L, Lazeyras F, Eliez S, Thiran JP (2008) A Surface-based approach to quantify local cortical gyrification. IEEE Trans Med Imaging 27:161-170 Available at: http://ieeexplore.ieee.org/document/4359040/ [Accessed February 28, 2018].

Schepers IM, Yoshor D, Beauchamp MS (2014) Electrocorticography Reveals Enhanced Visual Cortex Responses to Visual Speech. Cereb Cortex:4103-4110 Available at: http://www.ncbi.nlm.nih.gov/pubmed/24904069.

Schroeder CE, Lakatos P (2009) Low-frequency neuronal oscillations as instruments of sensory selection. Trends Neurosci 32:9-18 Available at: http://www.ncbi.nlm.nih.gov/pubmed/19012975 [Accessed March 13, 2018].

Schroeder CE, Lakatos P, Kajikawa Y, Partan S, Puce A (2008) Neuronal oscillations and visual amplification of speech. Trends Cogn Sci 12:106-113 Available at: http://www.ncbi.nlm.nih.gov/pubmed/18280772.

Schwartz JL, Savariaux C (2014) No, There Is No 150 ms Lead of Visual Speech on Auditory Speech, but a Range of Audiovisual Asynchronies Varying from Small Audio Lead to Large Audio Lag. PLoS Comput Biol 10.

Shah AS, Bressler SL, Knuth KH, Ding M, Mehta AD, Ulbert I, Schroeder CE (2004) Neural Dynamics and the Fundamental Mechanisms of Event-related Brain Potentials. Cereb Cortex 14:476-483.

Shannon R V, Zeng FG, Kamath V, Wygonski J, Ekelid M (1995) Speech recognition with primarily temporal cues. 270:303-304 Available at: http://www.ncbi.nlm.nih.gov/pubmed/7569981.

Sumby WH, Pollack I (1954) Visual Contribution to Speech Intelligibility in Noise. J Acoust Soc Am 26:212-215. 
Vander Ghinst M, Bourguignon M, Op de Beeck M, Wens V, Marty B, Hassid S, Choufani G, Jousmäki V, Hari R, Van Bogaert P, Goldman S, De Tiège X (2016) Left Superior Temporal Gyrus Is Coupled to Attended Speech in a Cocktail-Party Auditory Scene. J Neurosci 36:1596-1606 Available at: http://www.ncbi.nlm.nih.gov/pubmed/26843641 [Accessed January 3, 2019].

Zion Golumbic EM, Ding N, Bickel S, Lakatos P, Schevon CA, McKhann GM, Goodman RR, Emerson R, Mehta AD, Simon JZ, Poeppel D, Schroeder CE (2013) Mechanisms underlying selective neuronal tracking of attended speech at a "cocktail party". Neuron 77:980-991 Available at: http://dx.doi.org/10.1016/j.neuron.2012.12.037.

Zion Golumbic EM, Poeppel D, Schroeder CE (2012) Temporal context in speech processing and attentional stream selection: A behavioral and neural perspective. Brain Lang 122:151-161 Available at: http://dx.doi.org/10.1016/j.bandl.2011.12.010.

Zoefel B, Archer-Boyd A, Davis MH (2018) Phase Entrainment of Brain Oscillations Causally Modulates Neural Responses to Intelligible Speech. Curr Biol 28:401-408.e5 Available at: http://www.ncbi.nlm.nih.gov/pubmed/29358073 [Accessed April 10, 2018]. 